\title{
BNREL
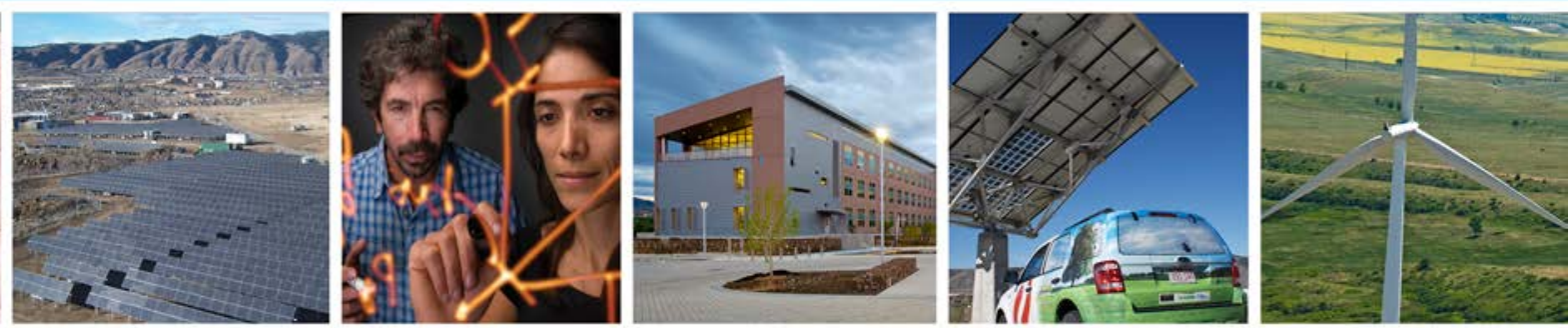

\section{Novel Approach for Calculation and Analysis of Eigenvalues and Eigenvectors in Microgrids}

\section{Preprint}

Y. Li, ${ }^{1,2}$ W. Gao, ${ }^{2}$ E. Muljadi, ${ }^{3}$ and J. Jiang ${ }^{1}$

${ }_{2}^{1}$ Beijing Jiaotong University

${ }^{2}$ University of Denver

${ }^{3}$ National Renewable Energy Laboratory

To be presented at the Clemson University Power Systems Conference

Clemson, South Carolina

March 11-14, 2014

NREL is a national laboratory of the U.S. Department of Energy Office of Energy Efficiency \& Renewable Energy Operated by the Alliance for Sustainable Energy, LLC

This report is available at no cost from the National Renewable Energy Laboratory (NREL) at www.nrel.gov/publications.

\section{Conference Paper}

NREL/CP-5D00-61255

February 2014 


\section{NOTICE}

The submitted manuscript has been offered by an employee of the Alliance for Sustainable Energy, LLC (Alliance), a contractor of the US Government under Contract No. DE-AC36-08G028308. Accordingly, the US Government and Alliance retain a nonexclusive royalty-free license to publish or reproduce the published form of this contribution, or allow others to do so, for US Government purposes.

This report was prepared as an account of work sponsored by an agency of the United States government. Neither the United States government nor any agency thereof, nor any of their employees, makes any warranty, express or implied, or assumes any legal liability or responsibility for the accuracy, completeness, or usefulness of any information, apparatus, product, or process disclosed, or represents that its use would not infringe privately owned rights. Reference herein to any specific commercial product, process, or service by trade name, trademark, manufacturer, or otherwise does not necessarily constitute or imply its endorsement, recommendation, or favoring by the United States government or any agency thereof. The views and opinions of authors expressed herein do not necessarily state or reflect those of the United States government or any agency thereof.

This report is available at no cost from the National Renewable Energy Laboratory (NREL) at www.nrel.gov/publications.

Available electronically at http://www.osti.gov/bridge

Available for a processing fee to U.S. Department of Energy and its contractors, in paper, from:

U.S. Department of Energy

Office of Scientific and Technical Information

P.O. Box 62

Oak Ridge, TN 37831-0062

phone: 865.576.8401

fax: 865.576 .5728

email: mailto:reports@adonis.osti.gov

Available for sale to the public, in paper, from:

U.S. Department of Commerce

National Technical Information Service

5285 Port Royal Road

Springfield, VA 22161

phone: 800.553 .6847

fax: 703.605.6900

email: orders@ntis.fedworld.gov

online ordering: http://www.ntis.gov/help/ordermethods.aspx 


\section{Novel Approach for Calculation and Analysis of Eigenvalues and Eigenvectors in Microgrids}

\author{
Yan $\mathrm{Li},{ }^{1,2}$ Member, IEEE \\ yanli2013power@gmail.com
}

\author{
Wenzhong Gao, ${ }^{2 *}$ Member, IEEE \\ Wenzhong.Gao@du.edu
}

\author{
Eduard Muljadi, ${ }^{3}$ Fellow, IEEE \\ eduard.muljadi@nrel.gov
}

\author{
Jiuchun Jiang, ${ }^{1}$ Member, IEEE \\ jcjiang@bjtu.edu.cn
}

\footnotetext{
${ }^{1}$ School of Electrical Engineering, Beijing Jiaotong University, Beijing, China

2 Department of Electrical and Computer Engineering, University of Denver, Denver, CO, USA

${ }^{3}$ National Renewable Energy Laboratory, Golden, CO, USA
}

\begin{abstract}
The calculation of eigenvalues and eigenvectors plays an important role in the stability analysis and optimal design of microgrids with multiple distributed energy resources. Microgrid systems are usually operated in various uncertain conditions. In this paper, a novel approach based on matrix perturbation theory is proposed for the calculation and analysis of eigenvalues and eigenvectors in a microgrid system. Rigorous theoretical analysis to solve eigenvalues and the corresponding eigenvectors for a system under various perturbations caused by fluctuations of irradiance, wind speed, or loads is presented. A computational flowchart is then proposed for the unified solution of eigenvalues and eigenvectors in microgrids, aimed toward obtaining eigenvalues and eigenvectors intuitively under different perturbations, which makes repeatedly solving an eigenvalue unnecessary. Finally, the effectiveness of the matrix perturbation-based approach in microgrids is verified by numerical examples on a typical low-voltage microgrid network.
\end{abstract}

Index Terms--Matrix perturbation, microgrids, distributed energy resources, eigenvalue, eigenvector

\section{INTRODUCTION}

Microgrid technology appears to be an increasingly attractive mechanism to facilitate the integration of distributed energy resources (DER) to increase the reliability of existing power systems, alleviate stress on transmission and distribution systems, and reduce environmental pollution [1][3]. A major challenge of microgrid technology is the optimal design, management, and control of DER units and system loads, in which the calculation and analysis of eigenvalues and eigenvectors (eigen-solutions) plays an essential role [4].

The main objective of a microgrid operated under various small changes is to ensure its small-signal stability. When a microgrid contains multiple DER units and various loads, it possesses complex small-signal stability characteristics because of different features of DER units and loads. Therefore, it is critical to analyze the small-signal stability of microgrids under various uncertain conditions [5], [6].

Generally, the operating point of a microgrid changes frequently. Thus, the eigenvalue problem needs to be solved repeatedly. Not only is this extremely tedious and time- consuming, but also it poses difficulties in studying the details of eigen-solutions' variations caused by different changes in a system. To overcome these limitations, a novel approach based on matrix perturbation theory (MPT) is proposed. MPT is an efficient method for the eigen-solutions calculation, aimed toward describing variations of a system's inherent properties under different perturbations [7]-[9]. The eigensolutions can be easily obtained with good accuracy for a system under perturbation with parameters. Therefore, there is no need to repeatedly solve the eigenvalue for a modified system. In addition, detailed analysis can be conducted only on critical eigen-solutions, regardless of whether a perturbation occurs in the outputs of the DER units or at the loads.

The remainder of this paper is organized as follows. Section II introduces the basic first-order perturbation theory for distinct, multiple, and close eigenvalues. Section III describes a perturbation analysis and computational flowchart of a microgrid system with various DER units. Section IV provides numerical examples that verify the effectiveness of the proposed approach. Conclusions are drawn in Section V.

\section{MATRIX PERTURBATION THEORY}

A finite-dimensional matrix pair $(A, B)$ is considered, where $A$ is a real asymmetric matrix and $B$ is a positive definite matrix. Then, the generalized eigenvalue problem of complex modes can be described by the following (1),

$$
\left\{\begin{array}{l}
A v_{i}=\lambda_{i} B v_{i} \\
u_{i}^{T} A=\lambda_{i} u_{i}^{T} B
\end{array}\right.
$$

where $\lambda_{i}$ is the $i^{\text {th }}$ generalized eigenvalue, $v_{i}$ and $u_{i}^{T}$ are the right and left generalized eigenvectors, respectively, satisfying the following orthogonal normalization conditions:

$$
\left\{\begin{array}{l}
u_{i}^{T} B v_{j}=\delta_{i j} \\
u_{i}^{T} A v_{j}=\delta_{i j} \lambda_{i}
\end{array}\right.
$$


where $\delta_{i j}$ is the Kronecker sign.

Theorem 1. If $\lambda_{i 0}$ is an eigenvalue of the original system $\left(A_{0}, B_{0}\right)$ with the multiplicity $m(m \geq 1)$, the eigensolutions of the perturbed system $\left(A_{0}+\varepsilon A_{1}, B_{0}+\varepsilon B_{1}\right)$ are given as follows [7]-[11]:

$$
\begin{aligned}
& \lambda_{i}=\lambda_{i 0}+\varepsilon \lambda_{i 1}+\sqrt[m]{\varepsilon^{m+1}} \lambda_{i 2}+\sqrt[m]{\varepsilon^{m+2}} \lambda_{i 3}+\cdots \\
& v_{i}=v_{i 0}+\varepsilon v_{i 1}+\sqrt[m]{\varepsilon^{m+1}} v_{i 2}+\sqrt[m]{\varepsilon^{m+2}} v_{i 3}+\cdots \\
& u_{i}^{T}=u_{i 0}^{T}+\varepsilon u_{i 1}^{T}+\sqrt[m]{\varepsilon^{m+1}} u_{i 2}^{T}+\sqrt[m]{\varepsilon^{m+2}} u_{i 3}^{T}+\cdots
\end{aligned}
$$

where $\varepsilon$ is the small perturbation parameter; $\lambda_{i 0}, v_{i 0}$, and $u_{i 0}^{T}$ are the eigen-solutions of the original system; $\lambda_{i 1}, v_{i 1}$, and $u_{i 1}^{T}$ are the first-order perturbations of eigen-solutions; and $\lambda_{i 2}, v_{i 2}$, and $u_{i 2}^{T}$ are the second-order perturbations of eigen-solutions, etc. Taking into account the accurate solutions obtained by the first-order perturbations, only the first-order perturbations of the eigen-solutions are analyzed in this paper. Further details about the second- and high-order perturbations can be found in [7]-[9].

According to the special distribution of eigenvalues-i.e., the value of the multiplicity $m-$ MPT can be introduced from three perspectives: MPT for distinct eigenvalues, MPT for multiple eigenvalues, and MPT for close eigenvalues [7], [11].

\section{A. MPT for Distinct Eigenvalues}

When all of the eigenvalues are separated from each other, the system will have a set of complete eigenvectors to span the whole space based on the Banach theory [9]. Therefore, the eigenvectors under perturbations can be expressed in the linear combination form of the eigenvectors in the original system. Then the first-order perturbations of the distinct eigenvalues and the corresponding eigenvectors can be expressed as follows. Further details can be found in [7]-[11].

$\lambda_{i 1}=u_{i 0}^{T} A_{1} v_{i 0}-\lambda_{i 0} u_{i 0}^{T} B_{1} v_{i 0}$

$v_{i 1}=\sum_{\substack{j=1 \\ j \neq i}}^{n}\left(\left(-u_{j 0}^{T} A_{1} v_{i 0}+\lambda_{i 0} u_{j 0}^{T} B_{1} v_{i 0}\right) /\left(\lambda_{j 0}-\lambda_{i 0}\right)\right) v_{j 0}$

$u_{i 1}^{T}=\sum_{\substack{j=1 \\ j \neq i}}^{n}\left(\left(-v_{j 0}^{T} A_{1}^{T} u_{i 0}+\lambda_{i 0} v_{j 0}^{T} B_{1}^{T} u_{i 0}\right) /\left(\lambda_{j 0}-\lambda_{i 0}\right)\right) u_{j 0}^{T}$

\section{B. MPT for Multiple Eigenvalues}

When any eigenvalues of multiplicity are found in the original system, this kind of system is known as a degenerate one. There are two important characteristics in this type of system: first, eigenvalues of multiplicity may be separated into distinct ones when a perturbation is introduced into the original system; second, mutations may occur to eigenvectors corresponding to the eigenvalues of multiplicity under a perturbation, which is caused by the arbitrariness of the selection of corresponding eigenvectors in the original system [7]-[9]. The first-order perturbations of eigenvalues of multiplicity and the corresponding eigenvectors can be expressed as follows. Further details can be found in [7]-[11]

$$
\begin{aligned}
& \lambda_{i 1}=\operatorname{eig}\left(U_{r 0}^{T} A_{1} V_{r 0}-\lambda_{r 0} U_{r 0}^{T} B_{1} V_{r 0}\right) \\
& x_{i 1}=\sum_{\substack{k=1 \\
k \neq r, \cdots, r+m-1}}^{n} \frac{\sum_{j=1}^{m} \alpha_{i j}\left(-u_{k 0}^{T} A_{1} v_{r 0}^{(j)}+\lambda_{r 0} u_{k 0}^{T} B_{1} v_{r 0}^{(j)}\right)}{\left(\lambda_{k 0}-\lambda_{r 0}\right)} v_{k 0}
\end{aligned}
$$

$$
y_{i 1}^{T}=\sum_{\substack{k=1 \\ k \neq r, \cdots, r+m-1}}^{n} \frac{\sum_{j=1}^{m} \beta_{i j}\left(-v_{k 0}^{T} A_{1}^{T} u_{r 0}^{(j)}+\lambda_{r 0} v_{k 0}^{T} B_{1}^{T} u_{r 0}^{(j)}\right)}{\left(\lambda_{k 0}-\lambda_{r 0}\right)} u_{k 0}^{T}
$$

where eig $\left(U_{r 0}^{T} A_{1} V_{r 0}-\lambda_{r 0} U_{r 0}^{T} B_{1} V_{r 0}\right)$ in (9) represents the eigenvalue of the matrix $U_{r 0}^{T} A_{1} V_{r 0}-\lambda_{r 0} U_{r 0}^{T} B_{1} V_{r 0} ; U_{r 0}^{T}$ and $V_{r 0}$ are the left and right eigenvector matrices corresponding to the eigenvalues of multiplicity $\lambda_{r 0} ; x_{i 1}$ and $y_{i 1}^{T}$ are the first-order perturbations of the right and left eigenvectors corresponding to $\lambda_{r 0}$; and $\alpha_{i j}$ and $\beta_{i j}$ are coefficients of which definitions and solutions can be found in [7].

\section{MPT for Close Eigenvalues}

In a system, sometimes eigenvalues may be distributed in a cluster manner even though they are distinct eigenvalues. In such a case, although the perturbed solutions of close eigenvalues and their corresponding eigenvectors can be obtained through the first-order perturbation theory for distinct eigenvalues, the calculation accuracy may not be satisfactory in some research problems. Therefore, it is necessary to investigate the special MPT for close eigenvalues.

Based on matrix spectrum decomposition theory, intensity index approach for eigenvalue identification, and Ritz base theory [7], [11], the original matrix $A_{0}$ can be rewritten in the following form:

$$
\begin{aligned}
A_{0}= & B_{0}\left[V_{P 0} \vdots V_{Q 0}\right] \operatorname{diag}\left(S_{M}, S_{Q 0}\right)\left[U_{P 0} \vdots U_{Q 0}\right]^{T} B_{0} \\
& +B_{0}\left[V_{P 0} \vdots V_{Q 0}\right] \operatorname{diag}\left(\delta S_{M 0}, 0\right)\left[U_{P 0} \vdots U_{Q 0}\right]^{T} B_{0} \\
= & \bar{A}_{0}+\delta A_{0}
\end{aligned}
$$

Where

$$
\left(S_{P 0}, S_{Q 0}\right)=(\underbrace{\lambda_{10}, \lambda_{20}, \cdots, \lambda_{\mathrm{p} 0}}_{S_{P 0}}, \underbrace{\lambda_{\mathrm{p}+1,0}, \cdots, \lambda_{n, 0}}_{S_{Q 0}})
$$




$$
\begin{aligned}
& {\left[V_{P 0} \vdots V_{Q 0}\right]=\left[v_{10}, v_{20}, \cdots, v_{\mathrm{p} 0} \vdots v_{\mathrm{p}+1,0}, \cdots, v_{n, 0}\right]} \\
& {\left[U_{P 0} \vdots U_{Q 0}\right]=\left[u_{10}, u_{20}, \cdots, u_{\mathrm{p} 0} \quad \vdots u_{\mathrm{p}+1,0}, \cdots, u_{n, 0}\right]} \\
& S_{P 0}=\left(\lambda_{m}+\delta \lambda_{10}, \lambda_{m}+\delta \lambda_{20}, \cdots, \lambda_{m}+\delta \lambda_{\mathrm{p} 0}\right) \\
& \quad=S_{M}+\delta S_{M 0} \\
& \lambda_{m}=\sum_{i=1}^{p} \lambda_{i 0} / p
\end{aligned}
$$

It is worth noting that there are two kinds of perturbations during the calculation process of the first-order perturbations of close eigenvalues: one is caused by the eigenvalues shift, e.g., $\delta A_{0}$ as shown in (12); the other is caused by parameters' perturbations, which is the same as in the distinct or multiple eigenvalues solution process.

\section{PERTURbation AnAlysis AND COMPUTATION FLOWCHART OF MICROGRIDS}

It is critical to research the small-signal stability of a microgrid. Through the comprehensive analysis of DER units, loads, and networks [12], [13], a microgrid can be described by a set of differential and algebraic equations (DAE) as shown in (18) [12], [13].

$$
\left\{\begin{array}{l}
\dot{x}=F(x, y, p) \\
0=G(x, y, p)
\end{array}\right.
$$

where $x \in R^{n}$ represent state variables, $y \in R^{m}$ represent algebraic variables, and $p \in R^{p}$ represent control variables.

The small-signal stability model of a microgrid can be expressed as (19), which is known as a state matrix and governs the stability feature of a microgrid.

$$
A_{S Y S}=F_{x}-F_{y} G_{y}^{-1} G_{x}
$$

where $F_{x}$ and $G_{x}$ are matrices of partial derivatives of state variables, and $F_{y}$ and $G_{y}$ are matrices of partial derivatives of algebraic variables.

When the output of DER units (e.g., PV and wind) fluctuates or the system load changes, the above-mentioned state matrix $A_{S Y S}$ will change accordingly. The corresponding variation $\Delta A_{S Y S}$ can be expressed as follows:

$$
\Delta A_{S Y S}=\Delta A_{S Y S, G}+\Delta A_{S Y S, L}+\Delta A_{S Y S 1, G L}
$$

where the total increment of the state matrix $\Delta A_{S Y S}$ includes three parts: $\triangle A_{S Y S, G}$ represents the first part of the matrix increment caused by the variations of the output of generation units only, $\triangle A_{S Y S, L}$ represents the second part of the increment caused by the fluctuations of the loads only, and $\triangle A_{S Y S, G L}$ represents the third part of the increment caused by both the generation units and system loads. All of their expressions are given as the following equations - with the condition that no change happens on the system structure, i.e., $G_{y 0}$ is a constant matrix.

$$
\begin{aligned}
\Delta A_{S Y S, G}= & \sum_{i=1}^{m} \Delta F_{x, i}-F_{y 0} G_{y 0}^{-1}\left(\sum_{i=1}^{m} \Delta G_{x, i}\right)-\left(\sum_{i=1}^{m} \Delta F_{y, i}\right) G_{y 0}^{-1} G_{x 0} \\
& -\left(\sum_{i=1}^{m} \Delta F_{y, i}\right) G_{y 0}^{-1}\left(\sum_{i=1}^{m} \Delta G_{x, i}\right) \\
\Delta A_{S Y S, L}= & \sum_{j=1}^{n} \Delta F_{x, j}-F_{y 0} G_{y 0}^{-1}\left(\sum_{j=1}^{n} \Delta G_{x, j}\right)-\left(\sum_{j=1}^{n} \Delta F_{y, j}\right) G_{y 0}^{-1} G_{x 0} \\
& -\left(\sum_{j=1}^{n} \Delta F_{y, j}\right) G_{y 0}^{-1}\left(\sum_{j=1}^{n} \Delta G_{x, j}\right) \\
\Delta A_{S Y S, G L} & -\left(\sum_{i=1}^{m} \Delta F_{y, i}\right) G_{y 0}^{-1}\left(\sum_{j=1}^{n} \Delta G_{x, j}\right) \\
& -\left(\sum_{j=1}^{n} \Delta F_{y, j}\right) G_{y 0}^{-1}\left(\sum_{i=1}^{m} \Delta G_{x, i}\right)
\end{aligned}
$$

where $m$ is the number of generation units with output changes and $n$ is the number of system loads with power changes.

Based on (20)-(23), the increment of the state matrix can be expressed in the form of a combination of different variations. This makes it easier to analyze the different impacts of different units on the system stability characteristics. When the state matrix changes as a result of the perturbations of the parameters caused by fluctuations of irradiance, wind speed, or loads, the increments of the eigenvalues and eigenvectors can then be calculated according to Section II.

For the computation of eigen-solutions, a calculation flowchart is also proposed. In Figure 1, Modeling and Initialization includes system components modeling, powerflow calculation, and system variables initialization, etc. Eigen-solutions Calculation is then conducted in the initial condition using the QR algorithm [8]. Subsequently, Variations are detected both from the generation side and load side. Based on the eigen-solutions and detection results, Modes are then classified into different categories of eigenvalues. 


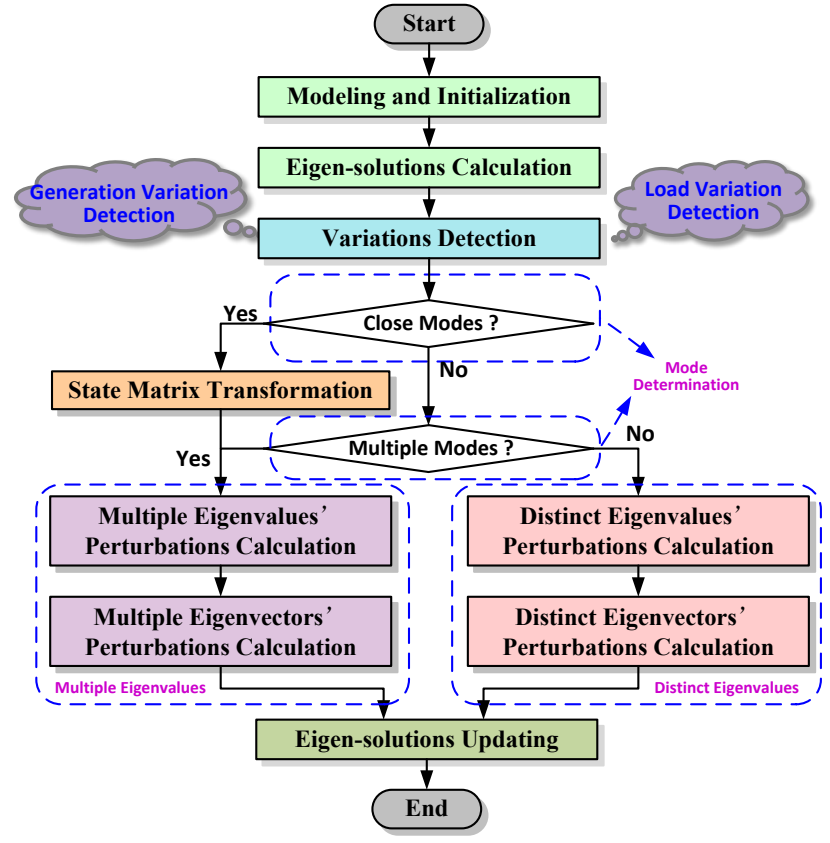

Figure 1. Flowchart of eigen-solutions based on MPT in a perturbed microgrid system

If there are any close modes in the original system, State Matrix needs to be transformed first, then the selection process of multiple modes can begin. If there are any multiple modes in the original system, Multiple Eigen-solutions Perturbation Calculation is carried out according to (9)-(11); or Distinct Eigen-solutions Perturbation Calculation is carried out according to (6)-(8). Finally, based on the first-order perturbations, eigen-solutions can be updated to analyze the new feature of small-signal stability in the perturbed system without repeatedly solving an eigenvalue problem.

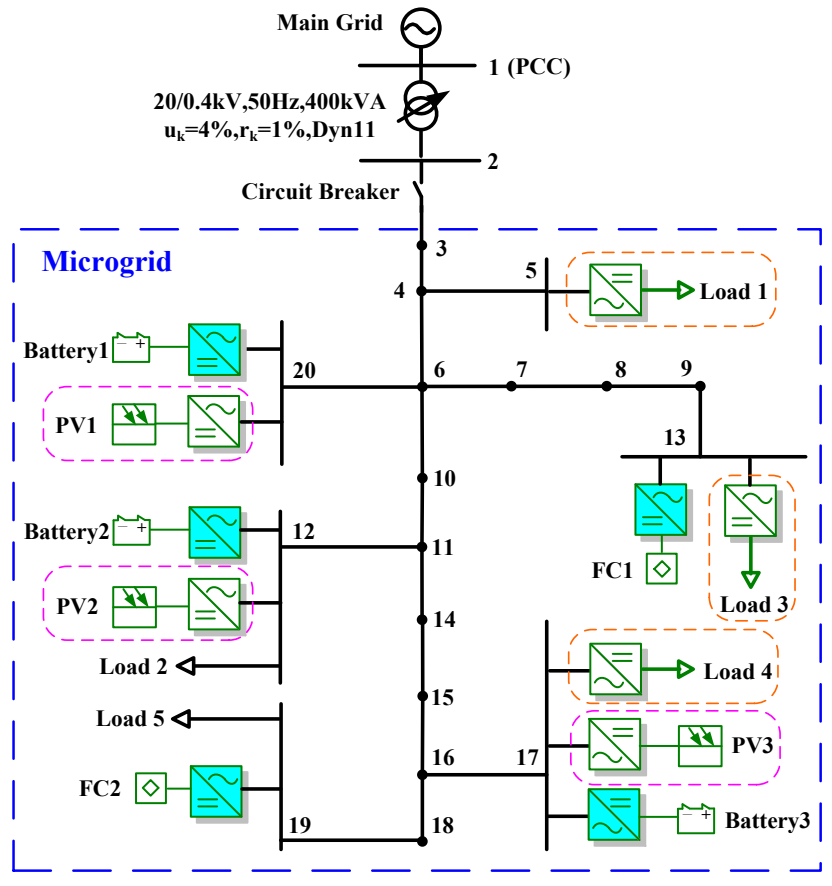

Figure 2. Typical low-voltage microgrid network

\section{NUMERICAL EXAMPLES}

The 0.4-kV, 50-Hz microgrid prototype shown in Figure 2 is used to test and verify the proposed approach. Parameters of this microgrid are provided in [14]. The numerical examples are carried out when the microgrid is operated in islanded mode and analyzed from the following two perspectives.

\section{A. Calculation and Analysis for Distinct and Multiple Eigenvalues}

There are 132 state variables in the test system. The multiple eigenvalues are given as follows: $-100.0,-9.75$, and -0.10 , with a multiplicity 6,2 , and 2 , respectively. Assuming that the irradiance of PV units and the power of loads are under different perturbations as shown in Table I, the solutions comparison of distinct eigenvalues and multiple ones between the MPT method and QR algorithm are shown in Figure 3 and Figure 4, respectively. Note that each perturbation is within the range of $\pm 10 \%$ for the computation accuracy.

TABLE I. PERTURBATIONS OF IRRADIANCE AND LOADS

\begin{tabular}{|c|c|c|c|c|}
\hline \multicolumn{2}{|c|}{} & Initial Value & Perturbation & Percentage \\
\hline \multicolumn{2}{|c|}{ Irradiance of PV1 $\left(\mathrm{W} / \mathrm{m}^{2}\right)$} & $1,000.00$ & 900.00 & $-10 \%(1)$ \\
\hline \multicolumn{2}{|c|}{ Irradiance of PV2 $\left(\mathrm{W} / \mathrm{m}^{2}\right)$} & $1,000.00$ & $1,050.00$ & $+5 \%(2)$ \\
\hline \multirow{2}{*}{ Load1 } & Active Power(W) & 12.75 & 14.00 & $+9.8 \%(3)$ \\
\cline { 2 - 5 } & Reactive Power(VAR) & 7.90 & 7.50 & $-5.1 \%(3)$ \\
\hline \multirow{2}{*}{ Load3 } & Active Power(W) & 61.15 & 65.00 & $+6.3 \%(4)$ \\
\cline { 2 - 5 } & Reactive Power(VAR) & 37.90 & 36.00 & $-5.0 \%(4)$ \\
\hline
\end{tabular}

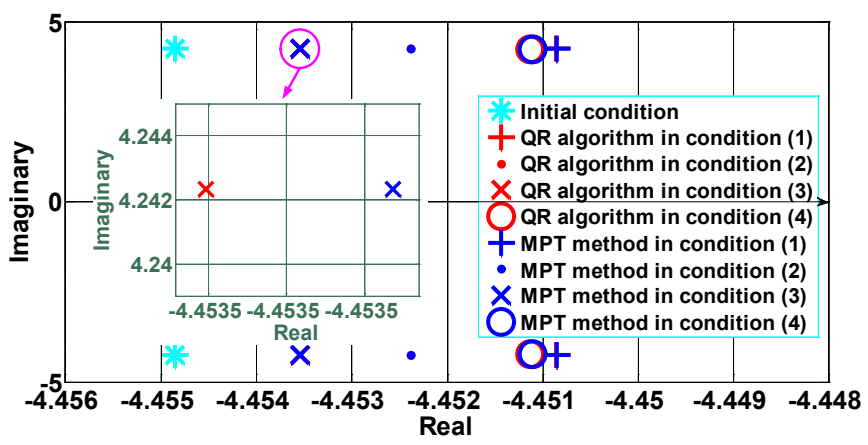

Figure 3. Comparison of distinct eigenvalues between the MPT approach and QR method under different perturbations

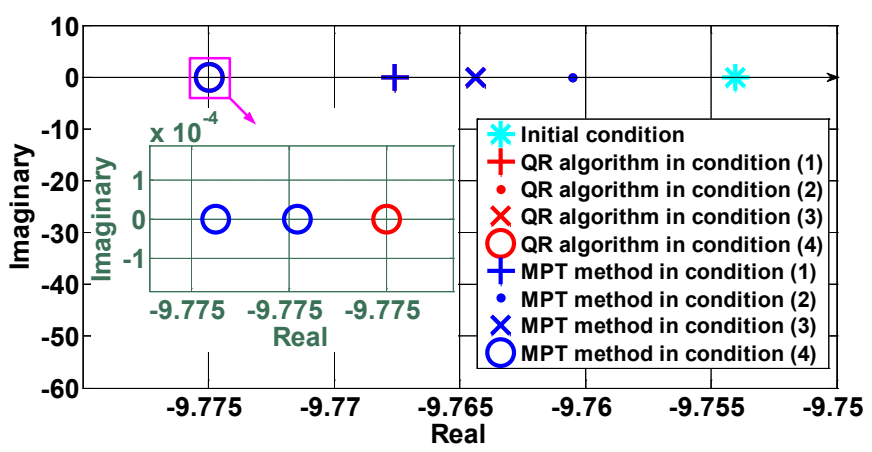

Figure 4. Comparison of multiple eigenvalues between MPT approach and QR method under different perturbations

Figure 3 shows only one conjugate of eigenvalues ($4.4548 \pm 4.2441$ i) for the comparison. The perturbed solutions are approximately equal to the corresponding exact ones 
within accuracy limits. A comparison of the multiple eigenvalues -9.75 is shown in Figure 4, which demonstrates the separation phenomenon of multiple eigenvalues during perturbations. Both of them validate the effectiveness of the MPT-based approach.

TABLE II. COMPARISON OF Multiple EIGENVAlues BetweEn THE MPT APPROACH AND QR METHOD

\begin{tabular}{|c|c|c|}
\hline & Perturbed Solutions & Exact Results \\
\hline \multirow{3}{*}{$\begin{array}{c}\text { Perturbation } \\
(1)\end{array}$} & $-102.7553+1.3178 \mathrm{i}$ & $-102.7555+1.3179 \mathrm{i}$ \\
\cline { 2 - 3 } & $-102.7002+1.2955 \mathrm{i}$ & $-102.7000+1.2953 \mathrm{i}$ \\
\cline { 2 - 3 } & $-102.6844+1.4568 \mathrm{i}$ & $-102.6844+1.4568 \mathrm{i}$ \\
\hline \multirow{3}{*}{$\begin{array}{c}\text { Perturbation } \\
(2)\end{array}$} & $-102.7538+1.3183 \mathrm{i}$ & $-102.7539+1.3183 \mathrm{i}$ \\
\cline { 2 - 3 } & $-102.7005+1.2942 \mathrm{i}$ & $-102.7004+1.2941 \mathrm{i}$ \\
\cline { 2 - 3 } & $-102.6844+1.4564 \mathrm{i}$ & $-102.6844+1.4564 \mathrm{i}$ \\
\hline \multirow{3}{*}{$\begin{array}{c}\text { Perturbation } \\
(3)\end{array}$} & $-102.7547+1.3181 \mathrm{i}$ & $-102.7548+1.3182 \mathrm{i}$ \\
\cline { 2 - 3 } & $-102.7010+1.2943 \mathrm{i}$ & $-102.7009+1.2942 \mathrm{i}$ \\
\hline \multirow{3}{*}{$\begin{array}{c}\text { Perturbation } \\
(4)\end{array}$} & $-102.6846+1.4561 \mathrm{i}$ & $-102.6846+1.4561 \mathrm{i}$ \\
\cline { 2 - 3 } & $-102.7553+1.3153 \mathrm{i}$ & $-102.7563+1.3156 \mathrm{i}$ \\
\cline { 2 - 3 } & $-102.7000+1.2978 \mathrm{i}$ & $-102.6991+1.2975 \mathrm{i}$ \\
\hline \multirow{2}{*}{\begin{tabular}{c} 
(4) \\
\cline { 2 - 3 }
\end{tabular}} & $-102.6865+1.4555 \mathrm{i}$ & $-102.6864+1.4555 \mathrm{i}$ \\
\hline
\end{tabular}

\section{B. Calculation and Analysis for Close Eigenvalues}

Close eigenvalues can usually be observed in a system due to the similar structure or parameter of units. When there is a long enough distance between the eigenvalues located in a cluster and the eigenvalues far away from this cluster, the eigen-subspace corresponding to this cluster is definitely a well-conditioned one.

In the test microgrid system, eight eigenvalue clusters can be found. Taking one of them as an example, the perturbed solutions and exact results are illustrated in Table II. The original eigenvalues of the cluster are given as follows: $102.7528+1.3195 \mathrm{i}, \quad-102.7019+1.2924 \mathrm{i}, \quad$ and $102.6843+1.4570 \mathrm{i}$.

As shown by the comparison of close eigenvalues in Table II, the effectiveness of the proposed method can be confirmed as well.

\section{CONCLUSION}

This paper proposes a novel method based on MPT for the calculation and analysis of eigen-solutions in a perturbed microgrid system. MPT for distinct, multiple, and close eigenvalues are introduced, respectively. Theoretical smallsignal stability analysis of microgrids is computed under perturbations of parameters caused by fluctuations of generation or load. The computational flowchart of the proposed method to solve perturbed microgrid system problems has also been presented. Numerical examples are performed in a typical low-voltage microgrid system. Theoretical analysis and evaluation results confirm the effectiveness of the MPT-based approach.

\section{ACKNOWLEDGMENTS}

This work was supported by the U.S. Department of Energy under Contract No. DE-AC36-08-GO28308 with the
National Renewable Energy Laboratory. This work is an activity performed under CRADA No. CRD11-427 between the National Renewable Energy Laboratory and the University of Denver. This work was also supported in part by NSF Grant 0844707 and ISTCP Grant 2013DFA60930.

\section{REFERENCES}

[1] R. H. Lasseter and P. Paigi, "Microgrid: A conceptual solution," in Proc. 2004 IEEE 35th PESC, pp. 4,285-4,290.

[2] J. A. P. Lopes, N. Hatziargyriou, and J. Mutale, "Integrating distributed generation into electric power systems: A review of drivers, challenges and opportunities," Electric Power Systems Research, vol. 77, pp. 1,189-1,203, 2007.

[3] R. H. Lasseter, J. H. Eto, B. Schenkman, J. Stevens, H. Vollkommer, D. Klapp, E. Linton, H. Hurtado, and J. Roy, "CERTS microgrid laboratory test bed," IEEE Trans. Power Del., vol. 26, pp. 325-332, Jan. 2011.

[4] X. Liu, P. Wang, and P. C. Loh, "A hybrid AC/DC microgrid and its coordination control," IEEE Trans. Smart Grid, vol. 2, pp. 278-286, Jun. 2011.

[5] E. A. A. Coelho, P. Cortizo, and P. F. D. Gracia, "Small-signal stability for parallel-connected inverters in stand-alone AC supply systems," IEEE Trans. Ind. Appl., vol. 38, no. 2, pp. 533-542, Mar./Apr. 2002.

[6] Z. X. Miao, A. Domijan, and L. L. Fan, "Investigation of microgrids with both inverter-interfaced and direct AC-connected distributed energy resources," IEEE Trans. Power Del., vol. 26, no. 3, pp. 1,6341,642, Jul. 2011.

[7] S. H. Chen, Matrix Perturbation Theory in Structural Dynamic Design. Beijing: Science Press, 2007, pp. 36, 132-137.

[8] J. H. Wilkinson, The Algebraic Eigenvalue Problem. Landon: Oxford University Press, 1965, pp. 62, 68-70.

[9] S. D. Assem, Advanced Matrix Theory for Scientists and Engineers. Kent: Abacus Press, 1982, pp. 205-215.

[10] C. S. Wang, Y. Li, K. Peng, B. W. Hong, Z. Wu, and C. B. Sun, "Coordinated optimal design of inverter controllers in a micro-grid with multiple distributed generation units," IEEE Trans. Power Sys., vol. 28, pp. 2,679-2,687, Aug. 2013.

[11] Y. Li, W. Z. Gao, J. C. Jiang, "Stability analysis of microgrids with multiple DER units and variable loads based on MPT," IEEE PES General Meeting 2014, to be published.

[12] Il-Y. Chung, W. X. Liu, D. A. Cartes, E. G. Collins, and Seung-Il Moon, "Control methods of inverter-interfaced distributed generators in a microgrid system," IEEE Trans. Ind. Appl., vol. 46, no. 3, pp. 1,0781,088, May./Jun. 2010.

[13] W. Huang, Z. P. Wu, M. Niu, J. H. Zhang, and C. Wu, "Dynamic modeling and simulation of microturbine generation system for the parallel operation of microgrid," IEEE International conference on sustainable power and supply, pp.1-8, Apr. 2009.

[14] S. Papathanassiou, N. Hatziargyriou, and K. Strunz, "A benchmark low-voltage microgrid network," in Proc. 2005 CIGRE Symposium Power Systems with Dispersed Generation, pp. 1-8. 